\title{
EDITORIAL
}

\section{The challenge of breathlessness in the detection of pulmonary hypertension}

\author{
F. Blasi
}

A cute or chronic shortness of breath (dyspnoea) is a common reason for patients to consult their primary care physicians. However, appropriate diagnosis and deciding whether or when to refer the patient to specialist care can be a challenge. Dyspnoea has been defined as "a subjective experience of breathing discomfort that consists of qualitatively distinct sensations that vary in intensity" [1]; however, patients may use a variety of descriptions for their symptoms, such as "my chest feels tight" or "I cannot get enough air" [2]. A patient's perception of their symptoms will also vary depending on their context, such as whether they occur on physical exertion or at rest, and on whether symptoms have come on acutely or developed gradually over time. Individuals with chronic dyspnoea are often limited in their activities, with resulting impairment in physical functioning and quality of life [1].

Dyspnoea can be a symptom of a variety of underlying conditions. These include cardiac conditions (such as myocardial infarction, arrhythmias or valvular disease), pulmonary conditions (such as asthma, chronic obstructive pulmonary disease (COPD), pneumonia, pulmonary embolism or lung malignancy), or a range of other causes (such as panic attacks, thyroid disease, anaemia or obesity) [1-3]. In many cases, dyspnoea may be due to multifactorial causes [4]. For some patients, the probable cause of dyspnoea may be obvious, such as exacerbation of known asthma, COPD or heart failure, but for others, particularly younger previously healthy individuals, a thorough diagnostic evaluation to establish the underlying cause is required.

A full clinical history and physical examination provide the first clues to the diagnosis of dyspnoea. For example, intermittent breathlessness with triggering factors may suggest asthma, while a history of heavy tobacco smoking and a productive cough may suggest COPD [2,3]. Features such as persistent inspiratory crackles and clubbing of the fingers provide evidence towards idiopathic pulmonary fibrosis [5], while chest pain during dyspnoea may be caused by coronary or pleural disease [3]. Standard lung function tests may be useful in the assessment of dyspnoea, helping to distinguish restrictive lung diseases from obstructive airway disease [1]. Blood tests can reveal anaemia or show erythrocytosis in

CORRESPONDENCE: F. Blasi, Cardio-Thoracic Department, University of Milan, IRCCS Fondazione Cà Granda Milan, via F. Sforza 35, Milan 20122, Italy. E-mail: francesco.blasi@unimi.it

PROVENANCE: Publication of this peer-reviewed article was supported by Bayer Pharma AG, Germany (article sponsor, European Respiratory Review issue 123). patients with advanced COPD [2]. If history and basic examination do not provide an obvious diagnosis, or if the patient is suffering from severe symptoms, referral for specialist respiratory or cardiac assessment will be necessary.

A number of tests may be conducted in the diagnosis of dyspnoea. Chest radiography may reveal lung parenchymal disease characteristic of COPD, chest wall abnormalities, malignancy or congestive heart failure. An ECG may reveal a disturbance of heart rate or rhythm, cardiac ischaemia, ventricular hypertrophy or pericardial disease [6]. Normal ECG findings, while not excluding a diagnosis of heart failure, may make this diagnosis less probable. Pulse oximetry and arterial blood gas analysis can confirm gas exchange abnormalities, which are commonly found in patients with interstitial lung disease or emphysema [1]. Specialist spirometry tests include measurement of the diffusing capacity of the lung for carbon monoxide, which is reduced in patients with diseases affecting the lung parenchyma or vascular abnormalities and may be elevated in conditions where there is an increased effective pulmonary blood volume, such as asthma or left-toright cardiac shunts. In addition, methacholine bronchoprovocation challenge can identify airway hyperreactivity. Cardiopulmonary exercise testing aims to quantify pulmonary gas exchange, ventilation and cardiac function, and is useful in cases where there is no apparent cause for dyspnoea or in patients who have multiple potential causes for their breathlessness [7]. High-resolution computed tomography (CT) scanning may also be useful for patients in whom the cause of dyspnoea is unclear, as it can help to diagnose interstitial lung disease or bronchiectasis [2, 8] and CT pulmonary angiography is increasingly used for the diagnosis of pulmonary embolism [9]. Echocardiography is the most useful test for diagnosing most cardiac causes of chronic dyspnoea, such as left ventricular dysfunction, valvular dysfunction or pericardial disease. An echocardiogram can also be used to estimate pulmonary artery systolic pressure and, more specifically, right ventricular size and function. If pulmonary vascular disease is suspected, echocardiography with Doppler flow studies can demonstrate elevated pulmonary artery pressures and resultant tricuspid regurgitation [10, 11]. As in all areas of medicine, there is a risk of misdiagnosis of the underlying cause of dyspnoea, and so regular reassessment and consideration of alternative diagnoses is crucial [12].

While most cases of chronic breathlessness can be attributed to conditions such as asthma, congestive heart failure, COPD, 
pneumonia, cardiac ischaemia, interstitial lung disease and psychogenic conditions $[4,13]$, it is important to remain vigilant for less common causes of breathlessness. Of these, one of the most serious is pulmonary hypertension (PH). Defined by an elevated resting mean pulmonary artery pressure $>25 \mathrm{mmHg}$, $\mathrm{PH}$ is a complex, multifactorial group of disorders that leads to progressive right ventricular failure [14-16]. As well as breathlessness, presenting symptoms include fatigue, weakness, angina pectoris, syncope and abdominal distension [17]. While very few patients with dyspnoea consulting their general practitioner or referred to the pulmonology outpatient clinic will be diagnosed with $\mathrm{PH}$, early diagnosis is so important that it must be always considered in the differential diagnosis. $\mathrm{PH}$ is not a single disease, but can be classified into five main categories depending on the mechanisms and underlying cardiovascular or respiratory condition: 1) pulmonary arterial hypertension (PAH); 2) $\mathrm{PH}$ due to left ventricular dysfunction (PH-LVD); 3) $\mathrm{PH}$ due to interstitial lung diseases and/or hypoxia (PH-ILD); 4) chronic thromboembolic pulmonary hypertension (CTEPH); and 5) $\mathrm{PH}$ with unclear and/or multifactorial mechanisms [14]. Of the categories of $\mathrm{PH}, \mathrm{PAH}$ has received the most research attention. All forms of $\mathrm{PH}$ are progressive and ultimately fatal; for example, untreated patients with PAH have an average survival of $<3$ yrs after diagnosis [18]. While the advent of pharmacological treatment for PAH has undoubtedly improved patients' symptoms and physical functioning, there are no approved treatments for the other types of $\mathrm{PH}$, and even for $\mathrm{PAH}$ current treatment strategies remain inadequate and the mortality rate continues to be high $[14,19,20]$. For example, in recently reported data from a French registry of PAH patients, 1-, 2- and 3-yr survival rates were 87, 76 and $67 \%$, respectively [21].

Diagnosis of all forms of $\mathrm{PH}$ at an early stage is important so that treatment may be started in order to delay disease progression before right heart failure develops. However, the rarity of the disease and the lack of symptoms specific to $\mathrm{PH}$ over other causes of breathlessness often thwart prompt diagnosis. Although $\mathrm{PH}$ is rare, it can strike relatively young patients who are otherwise healthy and in whom a serious disease would not normally be suspected. Delayed diagnosis is also attributable to clinical failure to recognise the combination of breathlessness and other symptoms as possibly related to $\mathrm{PH}$ and failure to fully evaluate symptoms. Consequently, misdiagnosis of $\mathrm{PH}$ as asthma is fairly common. In addition, patients may interpret an insidious onset of breathlessness and fatigue as being due to their lifestyle, being unfit or ageing, and so delay seeking medical attention until their daily activities are severely impaired [17]. Thus, diagnosis is often delayed by at least 2 yrs after the onset of symptoms [21, 22].

Once PAH is suspected from the patient's symptoms and results from an echocardiogram are available, definitive diagnosis of PAH requires accurate measurement of pulmonary artery pressure, pulmonary vascular resistance and cardiac output via right heart catheterisation [14]. While some clinicians rely on an echocardiogram to make a diagnosis of $\mathrm{PAH}$ because it is less invasive, it is necessary to demonstrate that the elevation in pulmonary artery pressure is due to increased pulmonary venous resistance, which can only be achieved by catheterisation [17]. It is also important to be aware that the resting physiological range of pulmonary artery pressure observed by echocardiography is dependent on age, sex and body mass index, with higher pressures often observed in older or obese individuals [10].

Underlying conditions that may be the cause of other types of $\mathrm{PH}$, such as thromboembolism, left ventricular dysfunction or pulmonary fibrosis, should be identified and treated. In particular, ventilation-perfusion scanning or spiral CT scanning of the chest may be used to confirm a diagnosis of CTEPH [2].

Despite the development of nine different drugs representing three classes of medication for the treatment of PAH over the past two decades, prognosis remains poor. In addition, no treatment has been approved for PH-LVD and PH-ILD and the only curative treatment for CTEPH is surgery, which is not suitable for many patients with the condition.

Ideally, therapy should slow down or reverse pulmonary vascular remodelling and preserve right ventricular function. It is generally understood that $\mathrm{PH}$ is a disorder of the pulmonary vasculature resulting in pathological vasoconstriction and proliferation of the vascular smooth muscle and endothelium. Current therapies for $\mathrm{PAH}$ target various signalling pathways known to be involved in vasoconstriction and proliferation, but these pathways are not yet fully elucidated. In addition, the associated conditions that result in the various types of $\mathrm{PH}$ are heterogeneous and sometimes do not seem to be related to one another, complicating comprehensive diagnosis and targeted treatment.

Another challenge is the accurate definition of $\mathrm{PH}$ phenotypes. Such definition may provide insights about the causes of disease, help to predict prognosis and ultimately identify which phenotypes are more likely to respond to a particular treatment or management strategy [23]. For example, one such phenotype is scleroderma-related $\mathrm{PAH}$, which carries a poorer prognosis compared with most other forms of PAH [24]. PAH appears to occur early in the course of scleroderma, and so not only are regular screening and prompt diagnosis required, but treatment strategies to target the relevant disease processes are urgently required [25]. Another more recently recognised $\mathrm{PH}$ phenotype is that of $\mathrm{PH}$ in association with combined pulmonary fibrosis and emphysema. This phenotype appears to have a particularly poor prognosis, with a recently reported 1 -yr survival rate of only $60 \%$ [26].

$\mathrm{PH}$ is a rare but serious group of conditions with no real cure. Despite the symptomatic improvement provided by current treatment strategies, there is much room for improvement in outcomes and many questions remain about the optimal management of $\mathrm{PH}$.

In this issue of the European Respiratory Review, several leading experts summarise the latest research on the pathophysiology of $\mathrm{PH}$ and discuss current management practices. WILKINS [27] discusses the science behind the disease spectrum of $\mathrm{PH}$, the understanding of which may one day provide targets for therapeutic intervention. VACHIÉRY et al. [28] describe current practices for detecting and monitoring disease progression in the clinic. The focus then shifts to CTEPH, with KIM and LANG [29] reviewing the risk factors behind the development of CTEPH, while JENKINS et al. [30] present the latest techniques for diagnosing CTEPH. 
I hope that you find this issue interesting and informative, and gain an understanding of the exciting new developments and ongoing research that provide hope in the future for improved outcomes for our patients with all types of $\mathrm{PH}$.

\section{STATEMENT OF INTEREST}

F. Blasi has received reimbursement for attending symposium, and fees for speaking, organising education and consultancy from AstraZeneca, Bayer, Boehringer, Chiesi, Diasorin, GSK, Nycomed, Pfizer and Zambon.

\section{ACKNOWLEDGEMENTS}

I received editorial assistance from Adelphi Communications (Bollington, UK), funded by Bayer Pharma AG (Berlin, Germany).

\section{REFERENCES}

1 Dyspnea. Mechanisms, assessment, and management: a consensus statement. American Thoracic Society. Am J Respir Crit Care Med 1999; 159: 321-340.

2 Karnani NG, Reisfield GM, Wilson GR. Evaluation of chronic dyspnea. Am Fam Physician 2005; 71: 1529-1537.

3 Zoorob RJ, Campbell JS. Acute dyspnea in the office. Am Fam Physician 2003; 68: 1803-1810.

4 Michelson E, Hollrah S. Evaluation of the patient with shortness of breath: an evidence based approach. Emerg Med Clin North Am 1999; 17: 221-237.

5 American Thoracic Society. Idiopathic pulmonary fibrosis: diagnosis and treatment. International consensus statement. American Thoracic Society (ATS), and the European Respiratory Society (ERS). Am J Respir Crit Care Med 2000; 161: 646-664.

6 Davie AP, Francis CM, Caruana L, et al. Assessing diagnosis in heart failure: which features are any use? QJM 1997; 90: 335-339.

7 American Thoracic Society, American College of Chest Physicians. ATS/ACCP statement on cardiopulmonary exercise testing. Am J Respir Crit Care Med 2003; 167: 211-277.

8 Hunninghake GW, Lynch DA, Galvin JR, et al. Radiologic findings are strongly associated with a pathologic diagnosis of usual interstitial pneumonia. Chest 2003; 124: 1215-1223.

9 Burns SK, Haramati LB. Diagnostic imaging and risk stratification of patients with acute pulmonary embolism. Cardiol Rev 2012; 20: 15-24.

10 Bossone E, Citro R, Blasi F, et al. Echocardiography in pulmonary arterial hypertension: an essential tool. Chest 2007; 131: 339-341.

11 Schiller NB. Pulmonary artery pressure estimation by Doppler and two-dimensional echocardiography. Cardiol Clin 1990; 8: 277-287.

12 Wills CP, Young M, White DW. Pitfalls in the evaluation of shortness of breath. Emerg Med Clin North Am 2010; 28: 163-181.

13 DePaso WJ, Winterbauer RH, Lusk JA, et al. Chronic dyspnea unexplained by history, physical examination, chest roentgenogram, and spirometry. Analysis of a seven-year experience. Chest 1991; 100: 1293-1299.
14 Galiè N, Hoeper MM, Humbert M, et al. Guidelines for the diagnosis and treatment of pulmonary hypertension: the Task Force for the Diagnosis and Treatment of Pulmonary Hypertension of the European Society of Cardiology (ESC) and the European Respiratory Society (ERS), endorsed by the International Society of Heart and Lung Transplantation (ISHLT). Eur Heart J 2009; 30: 2493-2537.

15 Rosenblum WD. Pulmonary arterial hypertension: pathobiology, diagnosis, treatment, and emerging therapies. Cardiol Rev 2010; 18: 58-63.

16 Rosenkranz S. Pulmonary hypertension: current diagnosis and treatment. Clin Res Cardiol 2007; 96: 527-541.

17 Houtchens J, Martin D, Klinger JR. Diagnosis and management of pulmonary arterial hypertension. Pulm Med 2011; 2011: 845864.

18 D'Alonzo GE, Barst RJ, Ayres SM, et al. Survival in patients with primary pulmonary hypertension. Results from a national prospective registry. Ann Intern Med 1991; 115: 343-349.

19 Benza RL, Miller DP, Gomberg-Maitland M, et al. Predicting survival in pulmonary arterial hypertension: insights from the Registry to Evaluate Early and Long-Term Pulmonary Arterial Hypertension Disease Management (REVEAL). Circulation 2010; 122: $164-172$.

20 Thenappan T, Shah SJ, Rich S, et al. Survival in pulmonary arterial hypertension: a reappraisal of the NIH risk stratification equation. Eur Respir J 2010; 35: 1079-1087.

21 Humbert M, Sitbon O, Chaouat A, et al. Survival in patients with idiopathic, familial, and anorexigen-associated pulmonary arterial hypertension in the modern management era. Circulation 2010; 122: 156-163.

22 Badesch DB, Raskob GE, Elliott CG, et al. Pulmonary arterial hypertension: baseline characteristics from the REVEAL Registry. Chest 2010; 137: 376-387.

23 Dweik RA, Erzurum SC. Update on pulmonary vascular diseases 2010. Am J Respir Crit Care Med 2011; 184: 26-31.

24 Chung L, Liu J, Parsons L, et al. Characterization of connective tissue disease-associated pulmonary arterial hypertension from REVEAL: identifying systemic sclerosis as a unique phenotype. Chest 2010; 138: 1383-1394.

25 Hachulla E, Launay D, Mouthon L, et al. Is pulmonary arterial hypertension really a late complication of systemic sclerosis? Chest 2009; 136: 1211-1219.

26 Cottin V, Le Pavec J, Prevot G, et al. Pulmonary hypertension in patients with combined pulmonary fibrosis and emphysema syndrome. Eur Respir J 2010; 35: 105-111.

27 Wilkins MR. Pulmonary hypertension: the science behind the disease spectrum. Eur Respir Rev 2012; 21: 19-26.

28 Vachiéry J-L, Yerly P, Huez S. How to detect disease progression in pulmonary arterial hypertension. Eur Respir Rev 2012; 21: 40-47.

29 Kim NH, Lang IM. Risk factors for chronic thromboembolic pulmonary hypertension. Eur Respir Rev 2012; 21: 27-31.

30 Jenkins D, Mayer E, Screaton N, et al. State-of-the-art chronic thromboembolic pulmonary hypertension diagnosis. Eur Respir Rev 2012; 21: 32-39. 